\title{
Bounds for bounded motion around a perturbed fixed point
}

By Ruud van Damme and Theo P. Valkering, Center for Theoretical Physics, University of Twente, $7500 \mathrm{AE}$ Enschede, The Netherlands

\section{Introduction}

Consider a mapping of the real plane onto itself

$$
x^{\prime}=H(x)+\xi, \quad x \in \mathbb{R}^{2} .
$$

$H(x)$ is a diffeomorphism, dissipative with positive constant Jacobian and has an attracting spiral. The extra term $\xi \in \mathbb{R}^{2}$ can represent a time dependent perturbation $\xi=\xi(x, n)$, bounded noise or a coupling to a second deterministic system $\left\{\xi=\xi(x, v), v^{\prime}=M(x, v)\right.$, with $\left.v \in \mathbb{R}^{n}\right\}$. Four our purpose the only and essential property is that it is bounded, i. e. there is a positive constant $\delta$ such that

$$
|\xi| \leqq \delta
$$

where $\|$ denotes the Euclidean norm in $\mathbb{R}^{2}$.

The aim of this paper is to predict the effect of the extra term $\xi$ on the attractor and its basin in dependence of $\delta$.

As an example we take for $H(x)$ the Henon mappirg (cf. $(2.1,13)$ ). In Fig. 1 a the basin of attraction is shown for one set of parameters values. In the presence of bounded noise with small enough amplitude, one expects a phase diagram as in Fig. $1 \mathrm{~b}$. Around the original attractor there is a region in which noisy orbits are captured. This set is called $A$. Around this set there is a region $B$ : all orbits starting in this region end up in $A$. There is a neighbourhood $C$ of the original basin boundary, where the asymptotic state of an initial condition depends on the particular noise sequence: orbits may converge to $A$, go to infinity or stay in $C$. Orbits starting outside $C$ always diverge. For increasing $\delta$, both $A$ and $C$ grow. At a critical value $\delta_{c}$ they touch and $B$ disappears, i.e. there are no initial conditions whose orbits remain bounded for all noise sequences $\{\xi\}$. In this paper a method is formulated to obtain a rigorous lower bound for $\delta_{c}$, and dependent on $\delta<\delta_{c}$ a "smallest" set around the origin which encloses $A$ and a "largest" set which is contained in $B$.

Our method relies upon the existence of a Lyapunov function in the basin of attraction of the spiral of $H(x)$, which can be constructed with arbitrary 

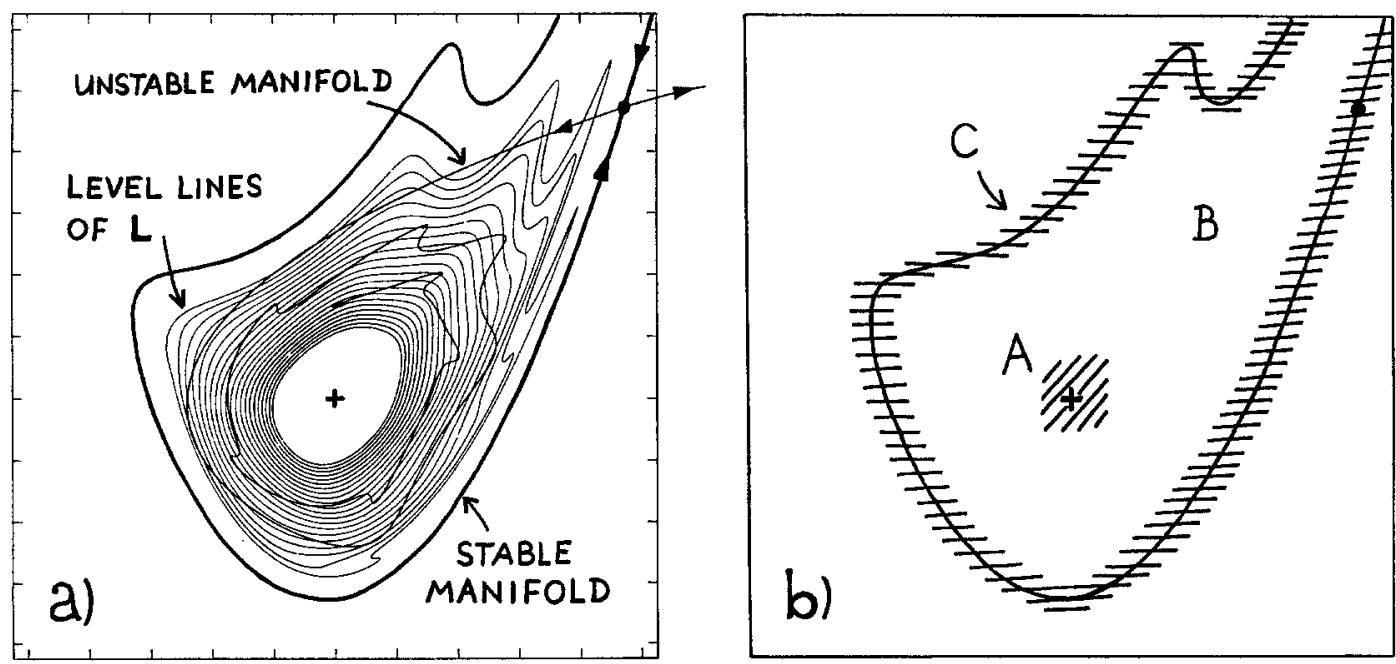

Figure 1

a) Sketch of the phase portrait for the Henon mapping $(2.1,13)$ at parametervalues $b=0.9$ and $c=0.3$. Shown are the attractor $(+)$ of $H(x)$ and the saddle $(\bullet)$ with its stable $\left(W^{s}\right)$ and unstable $\left(W^{u}\right)$ manifolds. $W^{s}$ is (part of) the boundary of the basin of the attractor. Orbits outside the region enclosed by $W^{s}$ diverge. Orbits within this region converge (but cf. Sect. 2). The closed contours are level lines of $L(x)$. Each level line is mapped by $H$ onto the nexts smaller one.

b) Sketch of the phase portrait of the full map (1.1) in case that $\xi$ represents bounded noise. Orbits starting near the fixed point of $H$ remain in $A$. Orbits starting in $B$ end up in $A$, and orbits starting in $C$ diverge or converge to $A$, dependent on the particular noise sequence.

accuracy for any mapping $H(x)$ with a constant positive Jacobian [1, 2]. This Lyapunov function $L(x)$ is defined everywhere in the basin of the attractor. It has a minimum equal to zero at the attractor and its level lines $C_{L}$ are closed contours around it, where $C_{L^{\prime}}$ encloses $C_{L}$ if $L<L^{\prime}$ (cf. Fig. 1 a). Convergence of an orbit to the attractor is governed by the relation $L(H(x))=\sqrt{b} L(x)$, where $b$ is the Jacobian of $H(x)$.

The estimates for $A$ and $B$ are based on comparison of the decrease of $L$ due to $H(x)$ and the possibly counteracting effect of the term $\xi$. In fact we demonstrate how to obtain estimates for a smallest $L_{a}$ and a largest $L_{b}$ such that the region $A$ is contained in $C_{L_{a}}$ and the interior of $C_{L_{b}}$ is contained in $B$. Each orbit starting between these two level lines will eventually end up in $C_{L_{a}}$.

In Sect. 2 the main elements related to the construction of the Lyapunov function are summarized, and details of a particular choice for $H$ (the Henon mapping) are given. The way to determine $L_{a}$ and $L_{b}$ is treated analytically in Sect. 3 and implemented numerically in Sect. 4. In Sect. 5 the resulting bounds are compared with the phase portrait of (1.1), where a particular (and unfavourable) choice for $\xi$ is used. 


\section{Properties of $H(x)$}

\subsection{The Lyapunov function for $H(x)$}

Let $H(x)$ in (1.1) have the form

$$
H(x)=P x+Q(x),
$$

where $P$ and $Q$ denote the linear and higher order terms respectively. The Jacobian is required to be a constant $b, 0<b<1 . P$ is supposed to have two complex eigenvalues, $\lambda$ and $\lambda^{*}$, such that $\lambda \lambda^{*}=b$, so that the origin is a spiral attractor.

First consider the linear map $x^{\prime}=P x$, which describes the approach of an orbit to the attractor at the origin asymptotically. Let $S$ diagonalize $P$ and define a matrix $A$ as follows

$$
A=S^{T} S, \quad S P S^{-1}=\operatorname{diag}\left(\lambda^{*}, \lambda\right) .
$$

With this definition one readily verifies

$$
(P x, A P x)=b(x, A x),
$$

where (,) denotes the usual innerproduct in $\mathbb{R}^{2}$. Now observe that the set

$$
E_{L}=\left\{x \mid(x, A x)=L^{2}\right\}
$$

is an ellipse since $A$ is symmetric and has positive eigenvalues. If $S$ is chosen such that det $S=1$, then $E_{L}$ encloses an area $\pi L^{2}$. Relation (2.3) then implies that the linear map $x^{\prime}=P x$ transforms the ellipse $E_{L}$ into a smaller ellipse $E_{L \sqrt{b}}$, which is just $E_{L}$ scaled with $\sqrt{b}$.

Thus $(x, A x)$ is a Lyapunov function for the linearization $P x$ of $H(x) . A$ Lyapunov function for the full nonlinear map, defined on the basin $B_{0}$, can be constructed as follows (for details we refer to [1]). There exists a real analytic transformation $x(u)$ with real analytic inverse $u(x)$, that maps the real $u$-plane onto $B_{0}$ and which satisfies

$$
H(x(u))=x(P u), u \in \mathbb{R}^{2} \quad P u(x)=u(H(x)), x \in B_{0} .
$$

This relation expresses the fact that each orbit of $u^{\prime}=P u$ is transformed by $x(u)$ to an orbit of $x^{\prime}=H(x)$ in $B_{0}$, and vice versa by $u(x)$. This transformation can be constructed with the aid of the relation

$$
x(u)=\lim _{n \rightarrow \infty} H^{-n}\left(P^{n} u\right) .
$$


A essential property is that $x(u)$ has Jacobian equal to +1 , so that it preserves area and orientation. For small $u$ it is approximately the identity, $x(u)=u+$ h.o.t.

With the aid of $u(x)$ we now define the functional $L$ in the basin $B_{0}$

$$
L(x)=(u(x), A u(x))^{1 / 2}, \quad x \in B_{0} .
$$

With (2.5) one now readily verifies the analogue of (2.3)

$$
L^{2}(H(x))=(P u(x), A P u(x))=b L^{2}(x), \quad x \in B_{0} .
$$

Since $L^{2}$ is nonnegative with only one critical point, a minimum at the attractor, (2.8) governs the convergence of an orbit in $B_{0}$ in a way similar to (2.3) in case of the linearised map. The level set $C_{L}$

$$
C_{L}=\left\{x \mid(u(x), A u(x))=L^{2}\right\}
$$

is mapped by $H(x)$ onto the level set $C_{L \sqrt{b}}$.

From the definition it is obvious that $C_{L}$ is the image of the ellipse $E_{L}$ by $x(u)$. Since $x(u)$ is area preserving, $C_{L}$ encloses an area equal to $\pi L^{2}$. Two ellipses $E_{L}$ and $E_{L^{\prime}}$ do not have points in common and $E_{L^{\prime}}$ encloses $E_{L}$ if $L<L^{\prime}$. Consequently the same statements hold for the contours $C_{L}$ and $C_{L^{\prime}}$, since $u(x)$ is invertible and preserves area and orientation. So we conclude:

Each level line is a closed contour around the origin. Two level lines with different $L$ cannot intersect and $C_{L}$ encloses $C_{L^{\prime}}$ if $L>L^{\prime}$.

The basin boundary can be considered as the limit of $C_{L}$ for $L->\infty$ in the following sense: for each $x$ on the boundary dist $\left(x, C_{L}\right)$ converges to zero if $L$ goes to infinity.

Figure 2

Schematic graph of $C_{k}$ and $C_{m}$. The shaded area is the $a$-neighbourhood of of $C_{k}$. For each $x$ in this neighbourhood it holds $L(x) \leqq m$, again because of the nested structure of the level lines (cf. after (2.9)). The equality holds precisely at those $x \in C_{k}, y \in C_{m}$ which realise the smallest distance.

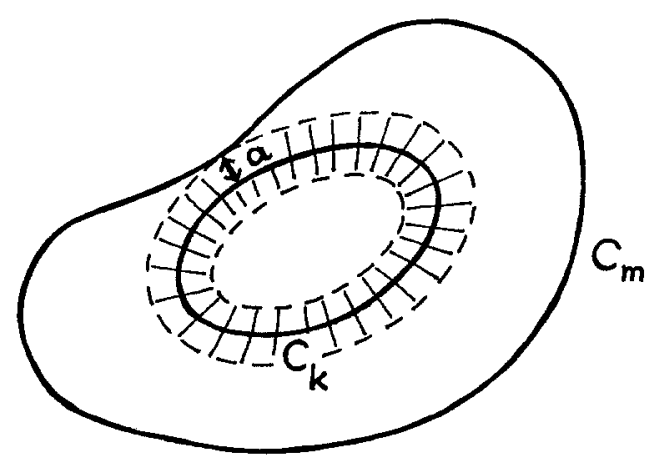


We now introduce two functions related to their distance. Let $a(k, m)$ denote the distance between $C_{k}$ and $C_{m}$, i.e. ( $k$ and $m$ are nonnegative real numbers)

$$
a(k, m)=\min |x-y| x \in C_{k}, y \in C_{m}, 0 \leqq k \leqq m k, m \in \mathbb{R} .
$$

Using the nested structure of the level lines one can immediately deduce that $a(k, m)$ increases as a function of $m$ for ficed $k$. Typically, i. e. unless the basin is the complete $\mathbb{R}^{2}, \lim _{m \rightarrow \infty} a(k, m)=a_{\infty}(k)$ exists and denotes the distance of $C_{k}$ to the basin boundary. Furthermore $a(k, m)$ decreases as a function of $k$ for fixed $m$, so that the inverse with respect to $m$ exists. This inverse is denoted by $m(k, a)$ and defined for $k \geqq 0$ and $0 \leqq a \leqq a_{\infty}(k)$. Again, if one observes that $C_{m}$ has a distance $a(k, m)$ to $C_{k}$, it is clear that $m(k, a)$ is an increasing function both of $k$ and of $a$. From Fig. 2 one can observe that $m(k, a)$ satisfies

$$
m(k, a)=\max L(y)\left|x \in C_{k},\right| x-y \mid \leqq a .
$$

Finally we define $d(k)$ which is the distance between $C_{k \sqrt{b}}$ and $C_{k}$

$$
d(k)=a(k \sqrt{b}, k)
$$

\subsection{The Henon mapping}

Our calculations are carried out for the Henon mapping, which we write as [3]

$$
P=\left[\begin{array}{cc}
2 c & -b \\
1 & 0
\end{array}\right], \quad Q(x)=\left(\begin{array}{c}
2 x^{2} \\
0
\end{array}\right)
$$

The origin is a spiral if $b>c^{2}$ with eigenvalues $\lambda$ and $\lambda^{*}$

$$
\lambda=c+i \sqrt{\left(b-c^{2}\right)} .
$$

The matrix $A$ the reads

$$
A=\left(b-c^{2}\right)^{-1}\left[\begin{array}{cc}
1 & -c \\
-c & b
\end{array}\right]
$$

The ellipses $E_{L}$ have their axes along the two eigenvectors of $A$. The ratio of their lengths equals $\alpha_{1} / \alpha_{2}$, in which $\alpha_{1,2}$ are the eigenvalues of $A$. 
The mapping has, apart from the spiral, another fixed point, a saddle at $x=(D, D)$, where

$$
D=(b+1) / 2-c .
$$

The stable manifold of this saddle forms, for the parameter values considered here, (part of) the boundary $B_{0}$. One way to construct such a manifold is described in [3]. If every orbit on the unstable manifold converges to the spiral, such as in Fig. 1 a, then $W^{s}$ is a nonfractal [5] (part of the) boundary of the basin. It is tempting to assume that the region of the plane bounded by $W^{s}$ forms the basin. This is not necessarily true, however. There can be other attracting periodic orbits (usually with very narrow basins) in this region as well.

\section{Determination of the bounds}

In this section it is shown how to determine a smallest $L_{a}$ such that $C_{L_{a}}$ encloses the "attractor" and a largest $L_{b}$ such that the region enclosed by $C_{L_{b}}$ is in the "basin". At the same time the critical bound $\delta_{c}$ is obtained.

Crucial to determine these quantities is the following observation. Consider the mapping (1.1) and let $x$ be on a level line $C_{L}$. Clearly its image $x^{\prime}$ is in a $\delta$ neighbourhood of $H(x)$. Thus we obtain immediately (cf. (2.11))

$$
L\left(x^{\prime}\right) \leqq m(\sqrt{b} L(x), \delta) \text { if } \delta<a_{\infty}(\sqrt{b} L),
$$

Thus the trajectories $\left\{L_{n}\right\}$ of the dynamical system defined by

$$
L^{\prime}=m(\sqrt{b} L, \delta), \quad L \geqq 0,
$$

form an upperbound on $\left\{L\left(x_{n}\right)\right\}$. A simple example where bounded motion occurs is shown in Fig. 3 a. Determination of the fixed points of (3.2) yields $L_{a}$ and $L_{b}$. The critical $\delta_{c}$ is precisely the smallest value of $\delta$ such that (3.2) has no fixed points.

To obtain these values one has to determine (numerically) the function $m(\sqrt{b} L, \delta)$ for the given $H(x)$. This is straightforward, but we will demonstrate now that essentially the same information can be obtained from $d(L)$, which is more simple since it depends on only one variable. Recall that $d(L)$ denotes (cf. (2.12)) the distance between two level lines $C_{\sqrt{b} L}$ and $C_{L}$. Its limiting values for small and large values of $L$ are known analytically

$$
\begin{aligned}
& d(L)=\beta L+O\left(L^{2}\right), \\
& \lim _{L \rightarrow \infty} d(L)=0 .
\end{aligned}
$$



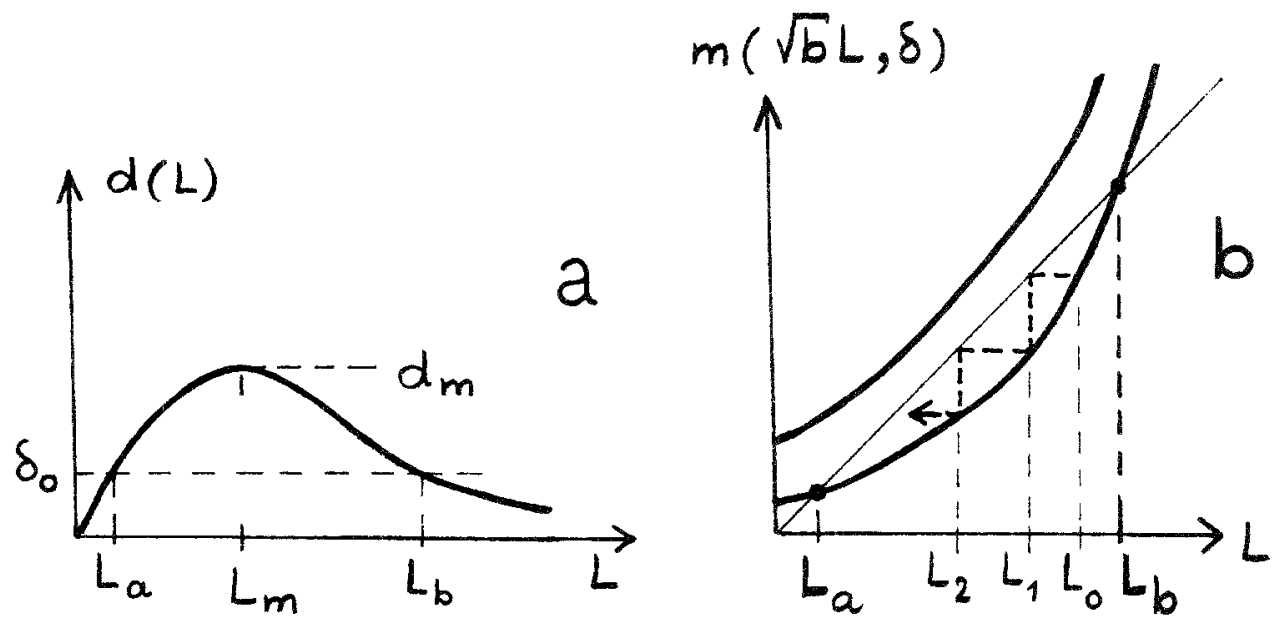

Figure 3

Graphs of $m(\sqrt{b} L, \delta)$ and of $d(L)$ in the simplest possible case. In a) also orbits of (3.2) are sketched. If $\delta<d_{m}$ orbits of (3.2) starting at $L_{a}<L_{0}<L_{b}$ converge monotonically to $L_{a}$.

Since for small $L$ the level lines $C_{L}$ are approximately the ellipses $E_{L}$ (cf. (2.4)), the term $\beta L$ represents the distance between two ellipses $E_{L}$ and $E_{\sqrt{b}}{ }_{L}$. Consequently the constant $\beta$ can be expressed in terms of the matrix $A$. In fact it holds

$$
\beta=(1-\sqrt{b}) \sqrt{\left(\alpha_{1} / \alpha_{2}\right)},
$$

where $\alpha_{1}$ and $\alpha_{2}$ are the eigenvalues of $A$, with $\alpha_{1}<\alpha_{2}$. Relation (3.3b) is a consequence of the fact that level sets $C_{L}$ accumulate on the basin boundary for $L \rightarrow \infty$. The most simple case is sketched in Fig. 3 b.

Now observe that the image of $x \in B_{0}$ is in a $\delta$-neighborhood of $H(x)$. Then since $H(x) \in C_{\sqrt{b} L}$ and because of the nested structure of the level lines $C_{L}$, it is obvious that

$$
\left.L\left(x^{\prime}\right)=L(H(x)+\xi)\right) \leqq L(x) \text { if } \delta \leqq d(L(x)) .
$$

Now consider Fig. 3 b. If $\delta<d_{m}, d(L)=\delta$ has two solutions, $L_{a}$ and $L_{b}$. Consequently points $x \in C_{L}$ are mapped onto points in the region enclosed by $C_{L}$ if $L_{a}<L<L_{b}$. One tentatively concludes that the orbits of such a point finally ends up in $C_{L_{a}}$. Such a conclusion, however, can be drawn in the base of (3.5) only by its relation to (3.1).

This relation is obtained in a straightforward manner. Since $m(k, \delta)$ is an increasing function of $\delta$, we have

$$
\delta \leqq d(L) \text { iff } m(\sqrt{b} L, \delta) \leqq m(\sqrt{b} L, d(L)), \text { for } 0 \leqq \delta \leqq a_{\infty}(L) .
$$

Because of the definition of $m$ and $d$ one readily sees that $m(\sqrt{b} L, d(L))=L$, so that we obtain

$$
\delta \leqq d(L) \quad \text { iff } \quad m(\sqrt{b} L, \delta) \leqq L .
$$



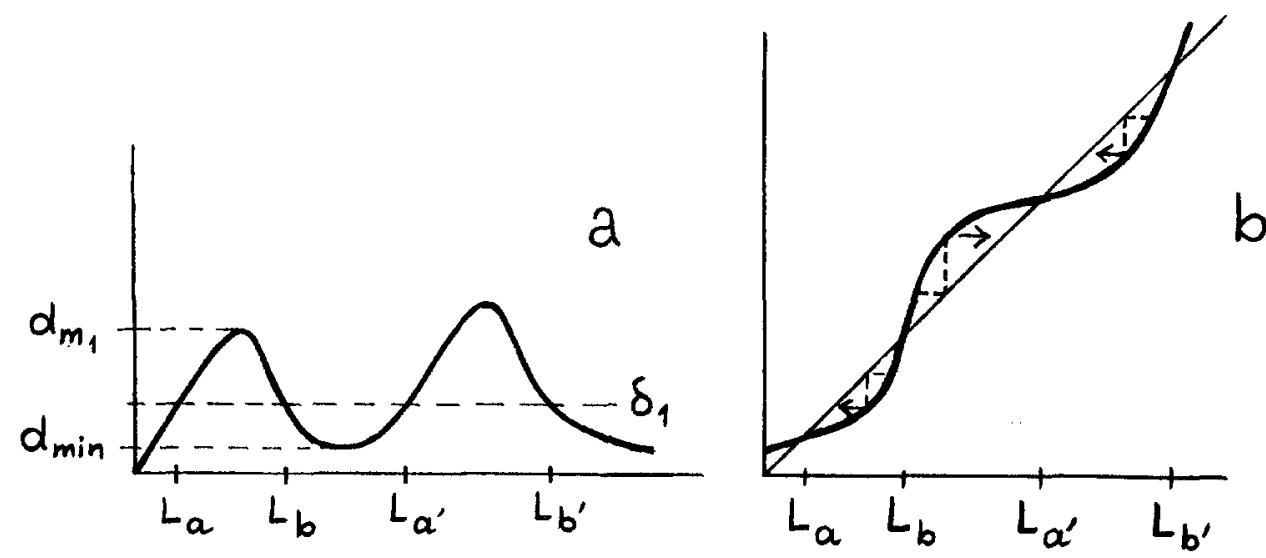

Figure 4

Possible graphs of $m(\sqrt{b} L, \delta)$ and of $d(L)$. In a) also orbits of (3.2) are sketched.

Now observe that one knows all about the relevant properties of the dynamical systems (3.2) if one knows the fixed points and the values of $L$ where $m(\sqrt{b} L, \delta)<L$. Consequently (3.7) demonstrates that it is sufficient to determine $d(L)$.

To interpret these results, consider the simple case of Fig. 3. For $\delta<d_{m}$ the dynamical system (3.2) has two fixed points: $L=L_{a}$ and $L=L_{b}$. A trajectory starting at $L_{0}<L_{a}$ increases monotonically and converges to $L_{a}$. A trajectory starting at $L_{a}<L_{0}<L_{b}$ decreases monotonically and also converges to $L_{a}$. If $L_{0}>L_{b}$ a trajectory diverges. Correspondingly for orbits of (1.1) we have

$$
\begin{array}{ll}
L\left(x_{n}\right) \leqq L_{n} \leqq L_{a} & \text { if } L\left(x_{0}\right)<L_{a}, \\
L\left(x_{n}\right) \leqq L_{n}->L_{a} & \text { if } L_{a}<L\left(x_{0}\right)<L_{b} .
\end{array}
$$

In words, all orbits of (1.1) starting between $C_{L_{a}}$ and $C_{L_{b}}$ converge to the area enclosed by $C_{L_{\alpha}}$. An orbit starting within $C_{L_{\alpha}}$ does not leave the enclosed area and nothing is known about orbits starting outside $C_{L_{b}}$. In the case of Fig. 4 similar conclusions can be drawn. Note however, that the only conclusion we can draw for orbits of (1.1) starting between $C_{L_{b}}$ and $C_{L_{a^{\prime}}}$ is that they remain within $C_{L_{a^{\prime}}}$. In practical cases it may well occur that these orbits also converge to $C_{L_{a}}$.

\section{Implementation of the method}

The method described in the previous Sections is implemented for the case that $H(x)$ is the Henon mapping. The parameters $c$ and $b$ are chosen to be fixed: $c=0.1$ and $b=0.7$. We discuss several ways to calculate $d(L)$. 
To calculate a level line $C_{L}$ one starts with an ellipse $E_{L}$ (2.4), applies $P n$ times and then $H^{-1}$ again $n$ times, with $n$ sufficiently large (cf. (2.6) and [1]). The distance function $d(L)$ can then be computed straightforwardly, searching the minimum of $|x-y|$ with $x \in C_{L}$ and $y \in C_{\sqrt{b} L}$.

This process to determine $d(L)$ is simple, in principle at least, but some practical problems arise, particularly for values of $L$ larger than $L_{m}$. In that case the level lines can have very long tentacles [1]. Consequently the number of pairs of points $x$ and $y$ one has to take grows disproportionally with $L$. Furthermore even if one starts with an even distribution of points on the initial ellipse $E_{L}$, the distribution of the resulting points on $C_{L}$ is very irregular. This makes the direct calculation of $d(L)$ time consuming and complicated.

To circumvent these difficulties we develop an approximate method, which uses a Taylor expansion in terms of $\delta$ of the right hand side of (3.2). It holds

$$
L^{2}(H(x)+\xi)=L^{2}(H(x))+\left(\xi, \nabla L^{2}(H(x))+1 / 2\left(\xi, \nabla \nabla L^{2}(H(x) \xi)+O\left(\xi^{3}\right) .\right.\right.
$$

Here we propose the following approximation for an upperbound of the right hand side. Define

$$
R(L)=\max _{x \in C \sqrt{b} L}|\nabla L(x)| .
$$

Then we clearly have

$$
L^{2}\left(x^{\prime}\right) \leqq b L^{2}(x)+\delta R(L)+1 / 2 \delta^{2} \sigma(H(x))+O\left(\delta^{3}\right),
$$

where $\sigma(x)$ denotes the largest eigenvalue of $\nabla \nabla L^{2}(x)$. An example of the behaviour of $|\nabla L(x)|$ along a leve line is shown in [5]. Let the maximum in (4.2) be attained at $x_{0}$. Then define

$$
S(L)=\sigma\left(x_{0}\right), \quad\left|\nabla L\left(x_{0}\right)\right|=R(L) .
$$

Now instead of (3.2) one can use trajectories of the dynamical process

$$
L^{2}=b L^{2}+\delta R(L)+\frac{1}{2} \delta^{2} S(L)
$$

as an upper bound for $L^{2}\left(x_{n}\right)$. Obviously, this upper bound is no longer rigorous. It can be improved if $\sigma$ (in (4.3)) is also maximized on $C_{\sqrt{b} L}$. However since $\nabla \nabla L^{2}(x)=A+O(x)$, this is not a large improvement for small values of $L$. For large values of $L$, the meaning of (4.5) is more doubtful because of the unknown effect of the omitted terms.

The analysis above is used in two ways. Firstly the distance between $x_{0}$ and $C_{L}$ is determined numerically. This function, denoted by $d_{0}(L)$ is shown in Fig. 5 a. Secondly we use (4.5) directly. To this end a function $\bar{d}(L)$ is introduced as the positive root of

$$
(1-b) L^{2}=\bar{d} R(L)+1 / 2 \bar{d}^{2} S(L),
$$



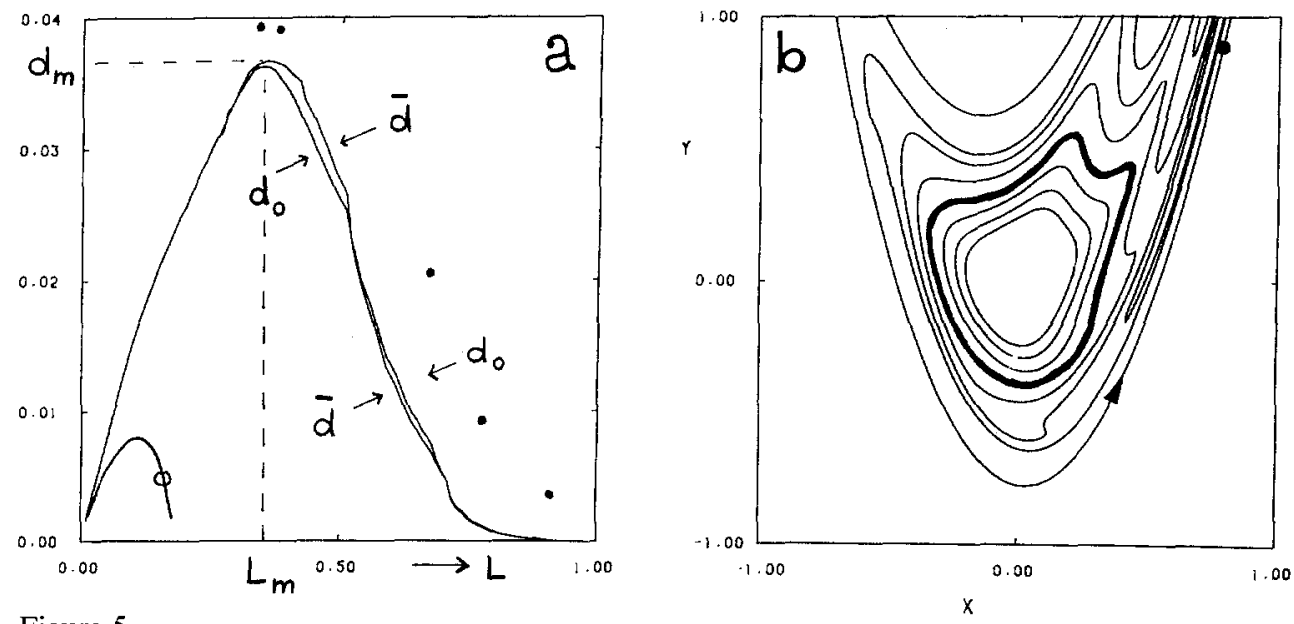

Figure 5

"Distance" functions $d_{0}(L)$ (cf. (4.2-4)) and $\bar{d}(L)$ for the Henon mapping at $b=0.7$ and $c=0.1$; $o$ denotes the distance function obtained with the quadratic norm $(x, A \mathrm{x})$. Some directly calculated values of $d(L)$ are indicated with $\bullet$. These graphs predict that there is bounded motion for $\delta<0.04$. Observe that there is almost no difference between $d_{0}$ and $\vec{d}$. For $L$ up to $\approx 0.9 L_{m}$ the calculated values of $d(L)$ coincide with $d_{0}(L)$, which is in agreement with the inequality $d_{0} \geqq d$. For $L$ bigger than that, however, these values exceed $d_{0}$ which seems to contradict this inequality. This demonstrates the difficulty in calculating $d$ as mentioned. In b) level lines at $L=b^{n / 2} L_{m}$ with $|n| \leqq 3$ are shown. The thick central line corresponds to the maximum $d_{m}$.

which is given

$$
\bar{d}(L)=\left(-R+\sqrt{ }\left(R^{2}-2 S(b-1) L^{2}\right)\right) / S .
$$

Clearly now the analogue of (3.7) holds

$$
\delta \leqq \bar{d}(L) \quad \text { iff } \quad L^{2} \geqq b L^{2}+\delta R(L)+\delta^{2} S(L) .
$$

Comparing $d_{0}(L)$ and $\bar{d}(L)$ in Fig. 5 , one observes that these curves almost coincide. For sake of comparison we also show in this graph a few values of $d(L)$ obtained by direct calculation as mentioned at the beginning of this Section. For $L<L_{m}$ this method yields value on de graphs $d_{0}(L)$ and $\bar{d}(L)$.

For the interpretation of the bounds found with $d_{0}(L)$ recall that, by its definition, $d(L) \leqq d_{0}(L)$. This implies that the values calculated with $d_{0}(L)$, indicated with a subscript $c$, always satisfy $L_{a c}<L_{a}, \delta_{c c}>\delta_{c}$ and $L_{b c}>L_{b}$.

The analysis above is based on the "best" Lyapunov function for the basin, incorporating fully the nonlinear aspects of the mapping. In principle one can apply the same method on the basis of the quadratic norm $(x, A x)^{1 / 2}$, related to the linearisation of the map. This however gives results which are inferior to those above. In that case one starts with an ellipse, $(x, A x)$ is constant, and determines the maximum value of $(H(x)+\xi, A(H(x)+\xi))$ for $\xi \leqq \delta$. In this way one obtains a relation similar to $(4.5)$, with $L$ representing the value of the quadratic norm. The resulting function distance function is shown in Fig. $5 \mathrm{a}$. 
We conclude that both $d_{0}(L)$ and $\bar{d}(L)$ are expected to yield useful approximations for $L_{a}, L_{b}$ and $\delta_{c}$. Values obtained with the quadratic norm are obviously inferior.

\section{Comparison with experiment}

Here we make a special, unfavourable, choice for $\xi$ and compare the values of $L_{a}, L_{b}$ and $\delta_{c}$ that are obtained in Sect. 4 with the results for this particular map. $H$ is the Henon mapping (2.13).

Write $\xi$ in (1.1) as

$$
\xi=\delta(\cos \varphi, \sin \varphi)
$$

and consider the mapping

$$
x^{\prime}=H(x)+\xi\left(\varphi_{0}\right),
$$

where $\varphi_{0}$ is chosen in such a way that

$$
L(H(x)+\xi(\varphi)) \leqq L\left(H(x)+\xi\left(\varphi_{0}\right)\right) \text { for } 0 \leqq \varphi \leqq 2 \pi,
$$

i. e. after each step $L$ is a large as possible within the restriction $|\xi| \leqq \delta$ (cf. (1.2)). Note that $\varphi_{0}$ is not necessarily unique for each $x$. In that case we pick one of the maximizing values at random.

First we test the meaning of $C_{L_{a}}$ and $C_{L_{b}}$ as obtained from Fig. $5 \mathrm{a}$. Choose $\delta=0.03$, somewhat smaller than the maximumvalue of $d$. To determine the region $A$, i. e. the "attractor" of the mapping, choose a set of initial points evenly distributed on the contour $C_{L_{a}}$. The orbits of these initial points all appear to end upon the contour $C_{A}$ shown in Fig. 6 . The same holds for orbits starting close to the origin. The value of $L(x)$ for $x \in C_{A}$ varies from $0.6 L_{a}$ to $0.7 L_{a}$. We conclude that $C_{L_{a}}$ gives a safe upper bound for the "attracting" set $A$.

Then, for the same value of $\delta$, choose initial conditions on radii $x=\alpha(\cos \psi$, $\sin \psi$ ). For fixed $\psi$ the smallest value of $\alpha$ for which the orbit does not converge to $C_{A}$ was determined. Doing this for several $\psi$ the contour $C_{B}$ shown in Fig. 6 was obtained. Actually every orbit within $C_{B}$ converges to $C_{A}$. Now $L(x)$ varies from $1.25 L_{b}$ to $1.5 L_{b}$ for $x \in C_{B}$. Consequently $C_{L_{b}}$ gives a safe lower bound for $C_{B}$

Secondly the largest value of $\delta$ such that there is bounded motion about the origin was obtained as follows. Let $a_{n}$ denote the $n^{\text {th }}$ iterate of the origin. Determine for given $\delta$ the smallest $N(\delta)$ such that $\left|a_{N}\right|$ is bigger than a suitably chosen large number $M$. Here $M$ is such that an orbit which comes outside the circle with radius $M$ runs of to infinity. The result was that orbits starting at the origin remain bounded for $\delta \approx<0.06$. Observe that this is well above the critical value 0.04 predicted by Fig. 5 . 
Figure 6

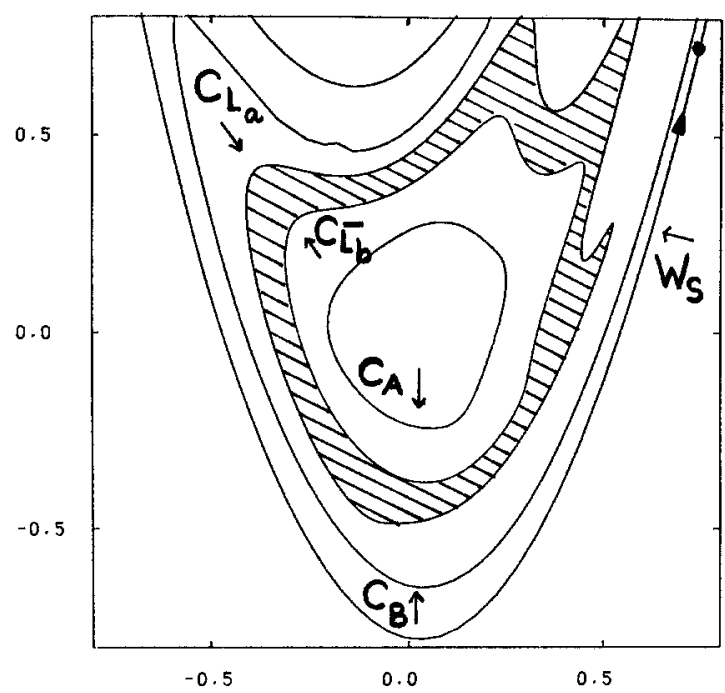

Comparison of the predictions in Sect. 4 with the actual orbits of the mapping (5.2) for $\delta=0.03$. The two bounds of the shaded area are $C_{L_{a}}$ and $C_{L_{b}}^{-}$, as obtained from Fig. 3. Each orbit in this area certainly ends up within $C_{L_{a}}$, i. e. independent of the choice for $\xi$. The contours $C_{A}$ and $C_{B}$ are obtained directly from the mapping (5.2). An orbit within $C_{A}$ converges to $C_{A}$. Each orbit within these two contours ends up on $C_{A}$. Outside $C_{B}$ there are orbits that diverge.

To conclude, the present method yields not very sharp but safe predictions for the "attracting set" $A$ near the attractor of the "undisturbed" system $x^{\prime}=H(x)$ and for the "basin of attraction" $B$. A safe lower bound is given for the maximally allowed value of the "perturbation" strength $\delta$, so that the extended system still has a stable "asymptotic state" containing the original one. In practice, in many cases, such as bounded noise, the decrease if $L$ at each step is bigger than the one as chosen in this Section. Consequently the predictions are less sharp but even safer. In the case of Gaussian noise the predictions are no longer rigorous. But one must expect that this method yields useful results as long as the terms do not exceed our critical value $\delta_{c}$.

\section{Acknowledgement}

One of us (RvD) is financially supported by the Stichting voor Zuiver Wetenschappelijk Onderzoek (ZWO) via the "Stichting Fundamenteel Onderzoek der Materie" (FOM).

\section{References}

[1] T. P. Valkering, The shape of the basin of attraction and transients via transformation to normal form. Physica 27D, 213-223 (1987).

[2] V. I. Arnold, Geometrical methods in the Theory of Ordinary Differential Equations (Springer, New York 1983). 
[3] R. van Damme and T. P. Valkering, Transient periodic behaviour related to a saddle-node bifurcation, J. Phys. A20, 4161-4171 (1987).

[4] R. H. G. Helleman, in: Long-time prediction in Dynamics, C. W. Horton, L. E. Reichl and A. G. Szebehely eds. (Wiley New York, 1983) p. 95.

[5] T. P. Valkering, Analytical description of the basin and the transients of a point attractor of the Henon mapping, Physica 18D, 483-485 (1986).

\begin{abstract}
We consider a dissipative map of the plane with a bounded perturbation term. This perturbation represents e.g. an extra time dependent term, a coupling to another system or noise. The unperturbed map has a spiral attracting fixed point. We derive an analytical/numerical method to determine the effect of the additional term on the phase portrait of the original map, as a function of the bound $\delta$ on the perturbation. This method yields a value $\delta_{c}$ such that for $\delta<\delta_{c}$ the orbits about the attractor are certainly bounded. In that case we obtain a largest region in which all orbits remain bounded and a smallest region in which these bounded orbits are captured after some time (the analogue of "basin" and "attractor" respectively).
\end{abstract}

The analysis is based on the Lyapunov function which exists for the unperturbed map.

(Received: May 6, 1988; revised: June 20, 1988) 